\title{
A minicourse on global existence and blowup of classical solutions to multidimensional quasilinear wave equations
}

\author{
Serge Alinhac
}

\begin{abstract}
The aim of this mini-course is twofold: describe quickly the framework of quasilinear wave equation with small data; and give a detailed sketch of the proofs of the blowup theorems in this framework.

The first chapter introduces the main tools and concepts, and presents the main results as solutions of natural conjectures. The second chapter gives a self-contained account of geometric blowup and of its applications to present problem.
\end{abstract}

\section{Introduction}

In this course, we deal only with multidimensional situations. Results and references on the analysis of small classical solutions for one dimensional systems may be found in Kong [21]. In chapter I, we present the three models we are considering, and explain a number of useful concepts. The results on the asymptotics of the lifespan are given at the end of this chapter. In chapter II, we focus on the proofs of upper bounds for the lifespans, trying to give a self-contained and unified account of results scattered in different papers.

In some sense, this course is an extension of my intoductory book [13]: the geometric blowup mechanism is more extensively studied, and the results on small solutions of quasilinear wave equations complete chapter $\mathrm{V}$ of the book, which contains only preliminary sketches.

MSC 2000 : 35L40.

Keywords : quasilinear wave equation, global existence, null condition, energy inequality, blowup, slowtime, Goursat problem. 


\section{Chapter I}

\section{SOME MODELS OF QUASILINEAR WAVE EQUATIONS IN TWO AND THREE SPACE DIMENSIONS: CONCEPTS AND RESULTS}

We consider here quasilinear wave equations in $\mathbb{R}_{t} \times \mathbb{R}_{x}^{n}$

$$
\left(\partial_{t}^{2}-\Delta_{x}\right) u+\sum_{0 \leq i, j \leq n} g_{i j}(u, \partial u) \partial_{i j}^{2} u=0 .
$$

Here $x_{0}=t, x=\left(x_{1}, \ldots, x_{n}\right)$, and $\partial u=\left(\partial_{1} u, \ldots, \partial_{n} u, \partial_{t} u\right)$. Unless otherwise indicated, indexes $i, j$ etc. in sums will range from 0 to $n$. We will also use the polar coordinates in $\mathbb{R}^{n}$ and the variable $\sigma$ defined by $r=|x|, x=r \omega, \omega_{0}=-1$, $\omega=\left(\omega_{1}, \ldots, \omega_{n}\right)$, and $\sigma=r-t$. The initial conditons for $u$ are

$$
u(x, 0)=\epsilon u_{1}^{0}(x)+\epsilon^{2} u_{2}^{0}(x)+\ldots, \quad \partial_{t} u(x, 0)=\epsilon u_{1}^{1}(x)+\epsilon^{2} u_{2}^{1}(x)+\ldots,
$$

where the real functions $u_{i}^{j}$ are smooth and supported in $|x| \leq M$.

The only case we consider where $g_{i j}$ depends on $u$ is the simplest case in $\mathbb{R}_{x}^{3}$

$$
\partial_{t}^{2} u-c^{2}(u) \Delta u=0, \quad c(u)=1+u
$$

In all other cases, $g_{i j} \equiv g_{i j}(\partial u)$, and the real smooth $g_{i j}$ verify

$$
g_{i j}(\xi)=\sum g_{i j}^{k} \xi_{k}+\sum h_{i j}^{k l} \xi_{k} \xi_{l}+O\left(|\xi|^{3}\right), \quad \xi \rightarrow 0
$$

We explain now some of the basic results and concepts needed to discuss our examples.

\section{Free solutions of the wave equation}

Following [16], we see that a solution $u$ of $\partial_{t}^{2} u-\Delta u=0$ with smooth compactly supported data (say, in $|x| \leq M$ ) can be approximately written

$$
u \sim r^{-\frac{n-1}{2}} F_{0}(\sigma, \omega)
$$

where $F_{0}$ is a smooth function which can be computed explicitly from the data, using Radon transform (the occurrence of Radon transform in this context can be easily seen by drawing backward light cones). For $n=2, F_{0}$ is a symbol in $\sigma$ of order $-1 / 2$, while it is supported in $|\sigma| \leq M$ for $n=3$ by Huyghens' principle. More precisely, we have for all $\alpha$ and $r \geq t / 2$

$$
\left|Z^{\alpha}\left(u-r^{-\frac{n-1}{2}} F_{0}\right)\right| \leq C(1+|\sigma|)^{(3-n) / 2}(1+t)^{-(n+1) / 2}
$$




\section{The $\mathrm{Z}$ fields}

We define, for $n=2$, the angle derivative $R=x_{1} \partial_{2}-x_{2} \partial_{1} \equiv \partial_{\omega}$. Similarly, for $n=3$, we define $R=x \wedge \partial$, that is

$$
R_{1}=x_{2} \partial_{3}-x_{3} \partial_{2}, \quad R_{2}=x_{3} \partial_{1}-x_{1} \partial_{3}, \quad R_{3}=x_{1} \partial_{2}-x_{2} \partial_{1} .
$$

These fields $R$ are tangent to the sphere $S^{n-1}$, and span the tangent space there. In polar coordinates, we have

$$
\begin{array}{cl}
\partial_{r}=\sum \omega_{i} \partial_{i}, \quad r \partial_{r}=x \partial_{x}, \quad \partial_{i}=\omega_{i} \partial_{r}-1 / r(\omega \wedge R)_{i}, & 1 \leq i \leq 3, \\
\Delta=\partial_{r}^{2}+1 / r \partial_{r}+1 / r^{2} \partial_{\omega}^{2}, & n=2, \\
\Delta=\partial_{r}^{2}+2 / r \partial_{r}+1 / r^{2} \Delta_{\omega}, \quad \Delta_{\omega}=R_{1}^{2}+R_{2}^{2}+R_{3}^{2}, & n=3 .
\end{array}
$$

We have the commutation relations $\left[R_{i}, \Delta\right]=0$ and $\left[\partial_{t}^{2}-\Delta, R_{i}\right]=0$. The other fields well commuting with the wave equation are

$$
\begin{aligned}
S & =t \partial_{t}+r \partial_{r}, & & {\left[\partial_{t}^{2}-\Delta, S\right]=2\left(\partial_{t}^{2}-\Delta\right), } \\
h_{i} & =t \partial_{i}+x_{i} \partial_{t}, & {\left[\partial_{t}^{2}-\Delta, h_{i}\right] } & =0 .
\end{aligned}
$$

The fields $R_{i}, S, h_{i}$ and $\partial_{j}$ are the $Z$ fields. Because of their homogeneity, the $Z$ fields are essentially connected with the zero cone $\{r-t=0\}: S$ and the hyperbolic rotations $h_{i}$ are only tangent to this cone; on the other hand, the rotations $R_{i}$ are tangent to all cones $\{r-t=C\}$.

Another aspect of the analysis is to consider, at each point, a special frame of vectors. To this aim, we introduce $T_{i}=\partial_{i}+\omega_{i} \partial_{t}, R / r=\omega \wedge T$. We define also

$$
L=\partial_{t}+\partial_{r}=\sum \omega_{i} T_{i}=(t+r)^{-1}\left(S+\sum \omega_{i} h_{i}\right), \quad \bar{L}=\partial_{t}-\partial_{r},
$$

and note that the tangent space to any cone $\{r-t=C\}$ is spanned by the $T_{i}$ or by $\left(R_{i}, L\right)$. On the other hand, the fields $T_{i}$ do not commute well with the wave equation. For more general geometries, analogous special vector fields are defined in [19]. The relations between the $Z$ fields and the $T_{i}$ are not simple:

$$
T_{i}=(1 / t) h_{i}-\omega_{i}(\sigma / t) \partial_{t}, \quad S=\sum x_{i} T_{i}-\sigma \partial_{t}
$$

Roughly, for $|\sigma| \leq C, T_{i}$ behaves like $(1 / t) Z$.

The following Klainerman's inequality explains the importance of the $Z$ fields:

$$
(1+|t|+|x|)^{n-1}(1+|| t|-| x||)|u(x, t)|^{2} \leq C \sum_{|\alpha| \leq n+2 / 2}\left|Z^{\alpha} u(., t)\right|_{L^{2}}^{2} .
$$

We note the double aspect of the decay property described by this inequality (assuming that the right-hand side is bounded):

1. For ||$t|-r|$ bounded (that is, close to the boundary of the light cone), $|u|$ decays like $t^{-\frac{n-1}{2}}$, 
2. When moving towards the interior of the light cone, the decay improve by a factor $t^{-1 / 2}$. This improvement is optimal for $n=2$, not for $n=3$.

The first aspect is easily understood if we think of using Sobolev inequalities for the variables $(r, \omega)$, for fixed $t$. The second aspect is more subtle, and uses the hyperbolic rotations $h_{i}$.

A typical way of gaining control of the right-hand side is by commuting products $Z^{\alpha}$ with the wave equation, and using the energy inequality.

In some situations, it is not possible to use all the fields $Z$. For instance, in elasticity theory, two different wave equations, with two different propagation speeds, occur: we can still use $S, R_{i}$, but no longer the hyperbolic rotations $h_{i}$ which depend on the speed. In compressible nonviscous fluid mechanics, the field $\partial_{t}+u . \nabla_{x}$ essentially governs the behavior of the vortex, and again, this prevents using the $h_{i}$. In [20], Klainerman and Sideris have found substitutes for Klainerman's inequality in these cases. These ideas have also been used by Sideris to study nonlinear elasticity [23].

\section{Energy inequalities}

There exists now a wide range of energy inequalities for perturbations of the wave equation. The definition of the standard energy of $u$ is

$$
E_{u}(t)=|(\partial u)(., t)|_{L^{2}}^{2} .
$$

The standard energy inequality is the following (see [16]).

Standard energy inequality. Let $u \in C^{2}$ satisfy the equation

$$
\left(\partial_{t}^{2}-\Delta\right) u+\sum g_{i j}(x, t) \partial_{i j}^{2} u=f, \quad 0 \leq t<T
$$

and assume $u(., t)$ has compact support. If $\sum\left|g_{i j}\right| \leq 1 / 2$, we have for $t<T$

$$
E_{u}(t)^{1 / 2} \leq 2\left(E_{u}(0)^{1 / 2}+\int_{0}^{t}|f(., s)|_{L^{2}} d s\right) \exp \left(2 \int_{0}^{t}\left|g^{\prime}(s)\right| d s\right)
$$

where

$$
\left|g^{\prime}(t)\right| \equiv \sum\left|\partial_{i} g_{j k}(., t)\right|_{L^{\infty}}
$$

This inequality is obtained by integrating by parts $f M u$ in the strip $\{0 \leq s \leq t\}$, for the multiplier $M u=u_{t}$. Note that the amplification factor $\exp \left(2 \int_{0}^{t}\left|g^{\prime}(s)\right| d s\right)$ involves all derivatives of the coefficients.

For the wave equation itself, it is possible to control directly the $Z$ derivatives of $u$, instead of $\partial u$. Defining a new energy

$$
\tilde{E}_{u}(t)=\sum|Z u(., t)|_{L^{2}}^{2}+|u(., t)|_{L^{2}}^{2}
$$


we have, for $n \geq 3$, the following Morawetz's type inequality

$$
\tilde{E}_{u}(t)^{1 / 2} \leq C \tilde{E}_{u}(0)^{1 / 2}+C \int_{0}^{t}(1+s)|f(., s)|_{L^{2}} d s
$$

This inequality is obtained by integrating $f M u$ as above, but this time for the (timelike) multiplier $M u=K_{0} u$, where $K_{0}=\left(r^{2}+t^{2}\right) \partial_{t}+2 r t \partial_{r}$.

For perturbations of the wave equation, similar inequalities can be found in [16] and [18]. Note that such inequalities are only available for $n \geq 3$, and that the control of the $Z$, which have linear coefficients, is obtained at the cost of an extra factor $(1+s)$ in the right-hand side.

In nonlinear problems, the coefficients $g_{i j}$ will be connected to the solution $u$, and one can assume that they behave similarly; in particular, one can assume that they can bear $Z$-fields. Such an appproach has been developped in [12], using the fields $T_{i}$ instead. Assuming (3.1) with $\sum\left|g_{i j}\right| \leq 1 / 2$ and $\sum\left|Z g_{i j}\right| \leq C(1+t)^{-\eta}$ for some $\eta>0$, we obtain for any $\epsilon>0$ and some $C_{\epsilon}$

$$
\begin{aligned}
E_{u}(t)+\int_{(0 \leq s \leq t)}(1+|\sigma|)^{-1-\epsilon} \sum\left(T_{i} u\right)^{2} d x d s & \\
& \leq C_{\epsilon} E_{u}(0)+C_{\epsilon} \int_{(0 \leq s \leq t)}|f|\left|u_{t}\right| d x d s+C_{\epsilon} \int_{0}^{t} A(s) E_{u}(s) d s .
\end{aligned}
$$

Here, the amplification factor $A$ is defined by

$$
A(t)=\left|\sum\left(\partial_{t} g_{i j}\right) \omega_{i} \omega_{j}\right|_{L_{x}^{\infty}}+\left|(1+|\sigma|)^{-1} \sum g_{i j} \omega_{i} \omega_{j}\right|_{L_{x}^{\infty}} .
$$

Compared with (3.2), we see that the amplification factor involves first order derivatives of the $g_{i j}$ only through the symmetric sums

$$
\sum\left(\partial_{t} g_{i j}\right) \omega_{i} \omega_{j}, \quad \sum g_{i j} \omega_{i} \omega_{j}
$$

We will see below that this may be important when cancellations occurs in such sums. Compared with (3.3), we obtain without extra cost a better control of the special derivatives $T_{i} u$. This is in accordance with what we know for free solutions, since

$$
T_{i}\left(r^{-\frac{n-1}{2}} F_{0}(\sigma, \omega)\right) \sim O\left(r^{-\frac{n+1}{2}}\right)
$$

actually behaves better than $\partial u$. In some sense, (3.4) is like (3.3) divided by $t$ on both sides, so to speak. Note also that (3.4) is true in any dimension.

When we later refer to Klainerman's energy method, we mean the following: to estimate the solutions of some perturbed wave equations, we commute products $Z^{\alpha}$ to the equation, and use one of the above energy inequalities. Finally, using Klainerman's inequality (2.1), one obtains pointwise decay of $u$. 


\section{The function $g(\omega)$ and the null condition}

For an equation (0.1) with $g$ satisfying (0.3), we define

$$
g(\omega)=\sum g_{i j}^{k} \omega_{i} \omega_{j} \omega_{k}
$$

We will see in 5 . how this function measures the strength of the quadratic nonlinearities. We will consider here three cases:

$$
\begin{array}{ll}
g(\omega) \not \equiv 0, & n=2, \\
g(\omega) \not \equiv 0, & n=3, \\
g(\omega) \equiv 0, & n=2 .
\end{array}
$$

When $g \not \equiv 0$, (case (1) or (2)), the solution $u$ will not exist globally in general. Precise statements will be given below. When $g \equiv 0$, we say, following Christodoulou and Klainerman, that the null condition is satisfied. They proved that in this case, if $n=3$, global smooth small enough solutions exist. The situation for $n=2$ is more complicated, since we have to take into account the cubic nonlinearities as well. Hence we define

$$
h(\omega)=\sum h_{i j}^{k l} \omega_{i} \omega_{j} \omega_{k} \omega_{l}
$$

A nontrivial two dimensional example where $g \equiv 0$ would be

$$
\partial_{t}^{2} u-\Delta u-\left(\partial_{1} u\right) \partial_{1 t}^{2} u+\left(\partial_{t} u\right) \partial_{1}^{2} u+a\left(\partial_{t} u\right)^{2} \Delta u=0
$$

In this case, $g \equiv 0$, and $h(\omega)=a$. It turns out that, if $h \not \equiv 0$, the solution $u$ will not exist globally in general. If again $h \equiv 0$ (which could be called the second null condition), then $u$ exists globally (see [9]).

In the above cases, when $u$ exists globally (that is, for an equation satisfying the null condition for $n=3$ or both null conditions for $n=2$ ), $u$ behaves essentially like a free solution:

$$
\left|Z^{\alpha} \partial u\right| \leq C \epsilon(1+t)^{-\frac{n-1}{2}}(1+|\sigma|)^{-\frac{n-1}{2}}
$$

This comes from Klainerman's method (for $n=2$ ) or (see [16]) from the use of some conformal transformation (for $n=3$ ). From this point of view, example (0.2) appears to be intermediate between the blowup cases (1) or (2), and these cases. In fact, we prove in [11] that the solution $u$ exists globally, and satisfies

$$
\left|Z^{\alpha} \partial u\right| \leq C \epsilon(1+t)^{-1+C \epsilon}(1+|\sigma|)^{-1 / 2} .
$$

We will see below that there is some evidence that the extra factor $(1+t)^{C \epsilon}$ is actually there: we say that this is an example of blowup at infinity. 


\section{The slow time}

A lot of insight into what happens to the equations (0.1) can be gained by trying to construct approximate solutions. Let us try a series

$$
u \sim \epsilon u^{(1)}+\epsilon^{2} u^{(2)}+\ldots
$$

We must take for $u^{(1)}$ the free solution with data $u_{1}^{0}, u_{1}^{1}$. From what we have seen in 1 ,

$$
u^{(1)} \sim r^{-\frac{n-1}{2}} F_{0}(\sigma, \omega)
$$

Next, $u^{(2)}$ is the solution of the Cauchy problem

$$
\begin{gathered}
\left(\partial_{t}^{2}-\Delta\right) u^{(2)}+Q=0, \quad Q=\sum g_{i j}^{k} \partial_{k} u^{(1)} \partial_{i j}^{2} u^{(1)} \\
u^{(2)}(x, 0)=u_{2}^{0}(x), \quad \partial_{t} u^{(2)}(x, 0)=u_{2}^{1}(x) .
\end{gathered}
$$

From the approximation of $u^{(1)}$ we get

$$
Q=r^{-(n-1)} g(\omega)\left(\partial_{\sigma} F_{0}\right)\left(\partial_{\sigma}^{2} F_{0}\right)+O\left(r^{-n}\right) .
$$

This is the origin of the function $g$ introduced in 4 . Hence the main term of $u^{(2)}$ behaves like $(\log t) / r$ for $n=3$ and $(t / r)^{1 / 2}$ for $n=2$. This gives

$$
\begin{array}{ll}
u=\epsilon r^{-1}\left(F_{0}+* \epsilon \log t+\ldots\right), & n=3, \\
u=\epsilon r^{-1 / 2}\left(F_{0}+* \epsilon t^{1 / 2}+\ldots\right), & n=2 .
\end{array}
$$

Thus we see that the terms of the series for $u$ stop being smaller and smaller when $\epsilon \log t$ ( respectively $\epsilon t^{1 / 2}$ ) is not small. This is the slow time effect, and we define the slow time of the problem by

$$
\tau=\epsilon \log t, \quad n=3, \quad \text { and } \quad \tau=\epsilon t^{1 / 2}, \quad n=2 .
$$

If $n=2$ and the equation satisfies the null condition, we see that the cubic nonlinearities acting on $u^{(1)}$ yield a term

$$
r^{-3 / 2} h(\omega)\left(\partial_{\sigma} F_{0}\right)^{2}\left(\partial_{\sigma}^{2} F_{0}\right),
$$

which is bigger than the bilinear term involving $u^{(1)}$ and $u^{(2)}$. Hence the main term in $u^{(3)}$ behaves like $(\log t) / r^{1 / 2}$ and this gives

$$
u=\epsilon r^{-1 / 2}\left(F_{0}+* \epsilon^{2} \log t+\ldots\right),
$$

displaying the slow time $\tau=\epsilon^{2} \log t$. 


\section{Reduced equations}

A step further in the analysis of 5 will be, considering the series expansions (5.1), to look for $u$ for large time as

$$
u \sim \epsilon r^{-\frac{n-1}{2}} G(r-t, \omega, \tau)
$$

$G$ being such that its Taylor expansion at time $\tau=0$ yields formally the series expansions obtained before. If we define $G$ by equality in (6.1), and write the equation on $u$ in terms of $G$, we obtain for cases (1) and (2)

$$
-\alpha_{n} \partial_{\sigma \tau}^{2} G+g(\omega)\left(\partial_{\sigma} G\right)\left(\partial_{\sigma}^{2} G\right)+O\left(\epsilon^{2}\right)=0, \quad \alpha_{2}=1, \quad \alpha_{3}=2,
$$

and, in case (3),

$$
-2 \partial_{\sigma \tau}^{2} G+h(\omega)\left(\partial_{\sigma} G\right)^{2}\left(\partial_{\sigma}^{2} G\right)+O\left(\epsilon^{2}\right)=0 .
$$

The notation $O\left(\epsilon^{2}\right)$ means here the product by $\epsilon^{2}$ of a second order operator in $(\sigma, \tau, \omega)$. We see that in all three cases the equation on $G$, for $\epsilon=0$, reduces to an equation that we call reduced equation. It is of Burgers'type for the unknown $\partial_{\sigma} G$. It is natural to solve the Cauchy problem for the reduced equations with datum

$$
G(\sigma, \omega, 0)=F_{0}(\sigma, \omega)
$$

We denote the corresponding solution by $G_{0}$.

If, in cases (1) or $(2), \max g(\omega) \partial_{\sigma}^{2} F_{0}>0$, the solutions $G_{0}$ have only a finite lifespan which is

$$
\bar{\tau}=\left(\alpha_{n} \max g(\omega) \partial_{\sigma}^{2} F_{0}\right)^{-1} .
$$

Similarly, in case $(3)$, if $\max h(\omega)\left(\partial_{\sigma} F_{0}\right)\left(\partial_{\sigma}^{2} F_{0}\right)>0, G_{0}$ has the finite lifespan

$$
\bar{\tau}=\left(\max h(\omega)\left(\partial_{\sigma} F_{0}\right)\left(\partial_{\sigma}^{2} F_{0}\right)\right)^{-1} .
$$

The same analysis as in case (2) can be carried out for the example (0.2). It turns out that the slow time $\tau=\epsilon \log t$ is the same, the reduced Cauchy problem being

$$
\partial_{\sigma \tau}^{2} G_{0}+G_{0} \partial_{\sigma}^{2} G_{0}=0, \quad G_{0}(\sigma, \omega, 0)=F_{0}(\sigma, \omega) .
$$

In contrast with the three cases considered above, it turns out that this Cauchy problem has a global solution $G_{0}$. However, $G_{0}$ and its derivatives satisfy

$$
\left|\partial_{\sigma} G_{0}\right| \leq C, \quad\left|\partial_{\sigma, \omega, \tau}^{\alpha} G_{0}\right| \leq C e^{C \tau}
$$

where the exponential growth actually occurs. This is why one can believe in global existence with blowup at infinity for equation (0.2).

Remark that the procedure explained in 5 and 6 gives a sort of algorithmic method to guess quickly what is likely to happen. It does not give a proof of longtime existence, or of blowup, but it is very handy. 
Let us finally mention the precise results obtained in case (1), (2) or (3). If the lifespan of $u$ (that is, the largest time until which $u$ stays $C^{\infty}$ ) is denoted by $\bar{T}_{\epsilon}$, we call in each case $\bar{\tau}_{\epsilon}$ this lifespan expressed in the variable $\tau$, that is

$$
\begin{array}{ll}
\bar{\tau}_{\epsilon}=\epsilon\left(\bar{T}_{\epsilon}\right)^{1 / 2} & \text { in case (1), } \\
\bar{\tau} \epsilon=\epsilon \log \bar{T}_{\epsilon} & \text { in case (2), } \\
\bar{\tau}_{\epsilon}=\epsilon^{2} \log \bar{T}_{\epsilon} & \text { in case (3). }
\end{array}
$$

Theoreme. Under the generic assumption (ND) on the data, we have in all three cases

$$
\lim _{\epsilon \rightarrow 0} \bar{\tau}_{\epsilon}=\bar{\tau}
$$

The assumption (ND) means that the maximum defining $\bar{\tau}^{-1}$ in (6.4) or (6.5) is unique, strictly positive and non degenerate. This theorem contains both

i) Large time existence theorems $\left(\lim \inf \bar{\tau}_{\epsilon} \geq \bar{\tau}\right)$,

ii) Blowup theorems $\left(\lim \sup \bar{\tau}_{\epsilon} \leq \bar{\tau}\right)$.

The large time theorems for case (1) and (2) have been proved by Hörmander [16]. For case (3), using the energy inequality (3.4), we see that the null condition induces cancellations in the amplification factor $A$ (see [9]). The blowup theorem for case (1) is proved in [4], [5], while [10] contains both case (2) and case (3).

In the next part, we give a detailed sketch of the proofs of the blowup theorems. It turns out that the very method we use also gives a precise description of the manner in which the solution blows up at time $\bar{T}_{\epsilon}$. The corresponding statements can be found in the already mentioned references.

\section{Chapter II \\ THE BLOWUP OF SMALL CLASSICAL SOLUTIONS IN THE GENUINELY NONLINEAR CASES}

We will sketch here the proofs of the blowup theorems of I. These proofs are rather long, and are based on the concept of geometric blowup. They offer two very different aspects:

1. An algebraic aspect, where various objects such as blowup system, blowup solutions, linearized blowup systems, etc. are introduced and discussed. "Algebraic" here does not refer to any theory, but describes simple manipulations with derivatives, change of variables, etc.

2. An analytic aspect, involving

a. The asymptotic behavior of the small solutions for large time,

b. The proof that the blowup systems (written in appropriate reduced variables), actually have smooth solutions. 
All the details and proofs are unfortunately scattered among different papers.

i) The algebraic aspects are first explained in [1], where also local existence of solutions to blowup systems is proved in the analytic case, using Cauchy Kowalevski theorem. To obtain smooth solutions to blowup systems, one has to analyze the corresponding linearized blowup systems: this is done in [5] for second order scalar equations (the case we need here), in [2] for 1D systems in a special case and in [8] for general multiD symmetric systems. This step is essential, since in applications it is practically impossible to write down explicitly these blowup systems, or (worse) to read from explicit formulas the geometric information we need. It turns out that the blowup system of a first order quasilinear symmetric system of size $N$ is a completely nonlinear first order system of size $N+1$, where the symmetry seems to be lost. It is only at the level of the linearized system that the symmetry can be restored. Parts $\mathrm{A}$ and $\mathrm{B}$ below give a self-contained account of these algebraic aspects.

ii) a) The analysis of the asymptotic behavior of the solutions for large time has been started in [16], where a rough approximate solution is constructed. We need here a somewhat more precise information, which amounts essentially to push the constructions of [16] to any order in $\epsilon$. The basic ideas of these nonlinear geometrical optics constructions are explained in [13], [16]. It turns out that the description of what happens close to the boundary of the light cone, which is all we need here, can be obtained quicker by a local argument using the $Z$ fields and the equation again. This argument is explained in C.1, referring to [10] for all the details. This allows us to reduce the blowup problem to a problem on a compact domain in the variables $(\sigma, \omega, \tau)$. In part C.2, we explain why this problem is a free boundary problem, and how it can be reduced, through some algebraic manipulations, to a problem on a fixed domain (the details can be found in [4], [5], [10]). At this stage, we need a certain stability of the way the solution is going to blowup: this is our condition $(\mathrm{H})$, which is itself a consequence of our assumption (ND) (see the end of chapter I).

b) Finally, part $\mathrm{D}$ is devoted to prove tame estimates ( in the sense of the Nash-Moser theorem we use here, see for instance [14]) for the linearized blowup systems. The fact is that these systems are degenerate precisely at points corresponding to blowup points: the measure of this degeneracy is given by $(\mathrm{H})$. For the general case $n=2$, details are given in [4], [5]. For the other cases, see [10].

\section{A. GEOMETRIC BLOWUP}

\section{A.1. Focusing and blowup in one space dimension}

Consider Burgers'equation

$$
\partial_{t} u+u \partial_{x} u=0, \quad x \in R
$$


with some initial data $u(x, 0)=u_{0}(x)$. If there exists a $C^{1}$ solution $u$ in a strip $0 \leq t<T$, the integral curves of the field $Z=\partial_{t}+u \partial_{x}$ are straight lines, since $Z u=0$ implies that $u$ is constant along each such curve. Hence the curve (called characteristic) starting from $\left(x_{0}, 0\right)$ is the line $x=x_{0}+t u_{0}\left(x_{0}\right)$, and all these curves are known from $u_{0}$ only. The solution is implicitely determined by

$$
u(x, t)=u_{0}(x-t u(x, t)) .
$$

For example, if $u_{0}$ is 1 for $x \leq-1,-1$ for $x \geq 1$ and $-x$ inbetween, all characteristics starting from $\left(x_{0}, 0\right),-1 \leq x_{0} \leq 1$ meet at $(0,1)$, where $u_{x}$ becomes infinite: this phenomenon is called focusing. A less degenerate example is obtained for $u_{0}$ smooth and decreasing, being 1 for $x \leq-2,-1$ for $x \geq 2$, 0 at 0 , with a non degenerate minimum of $u_{0}^{\prime}$ at $x=0$ with value -1 . Then the characteristics "almost meet" at $(0,1)$, in the sense that the mapping $x_{0} \mapsto x_{0}+u_{0}\left(x_{0}\right)$ has values $O\left(x_{0}^{3}\right)$ near $x_{0}=0$. Again in this case, $u_{x}$ becomes infinite at $(0,1)$.

Along each characteristic, $Z\left(u_{x}\right)+\left(u_{x}\right)^{2}=0$, hence

$$
u_{x}=\frac{u_{0}^{\prime}\left(x_{0}\right)}{1+t u_{0}^{\prime}\left(x_{0}\right)} \text {. }
$$

If $u_{0}^{\prime}\left(x_{0}\right)<0, u_{x}$ becomes infinite at time $t^{-1}=-u_{0}^{\prime}\left(x_{0}\right)$. For $X$ close to such a point $x_{0}$, let us consider the locus of these blowup points

$$
\gamma=\left\{(x, t), \quad x=X+s u_{0}(X), \quad s^{-1}=-u_{0}^{\prime}(X)\right\} .
$$

In the case $u_{0}^{\prime \prime}\left(x_{0}\right) \neq 0, \gamma$ is a smooth curve near $m_{0}$ (the point corresponding to $X=x_{0}$ ), and the solution $u$, defined on one side of $\gamma$, is singular all along $\gamma$ : we call this situation a fold singularity of $u$.

In the case $u_{0}^{\prime \prime}\left(x_{0}\right)=0, u_{0}^{\prime \prime \prime}\left(x_{0}\right)>0$, the curve $\gamma$ has a cusp point at $m_{0}$ (pointing downward), the solution is defined below $\gamma$ and is singular only at $m_{0}$ : we call this situation a cusp singularity. In both cases, $\gamma$ is an envelope of the characteristics.

We can now rephrase the method of characteristics explained above by defining

$$
\phi(X, T)=X+T u_{0}(X), \quad \Phi(X, T)=(\phi(X, T), T) .
$$

We see that $u(\Phi(X, T))=u_{0}(X)$, and the image of the vertical line $\left\{X=X_{0}\right\}$ is the characteristic starting from $\left(X_{0}, 0\right)$. In other words, the map $\Phi$ just straightens out, or defocuses, the characteristics. Also, $\gamma$ is the image by $\Phi$ of the set of points $(X, T)$ where $\Phi^{\prime}$ is not invertible. The two cases "fold" and "cusp" correspond respectively to the cases where the mapping $\Phi$ has at the point corresponding to $m_{0}$ a fold or a cusp singularity in the classical sense (see also $\mathbf{3 . 2}$ below). For instance, suppose that, close to $x=0, u_{0}$ is

$$
u_{0}(x)=\alpha-x / \bar{T}+\beta x^{3}, \quad \beta>0 .
$$

Then $x=0$ is a nondegenerate minimum of $u_{0}^{\prime}$, and $u_{x}$ blows up along the characteristic from $(0,0)$ at time $\bar{T}$. We find

$$
\phi(X, T)=\alpha T+\frac{\bar{T}-T}{\bar{T}} X+\beta T X^{3}, \quad \phi_{X}=\frac{\bar{T}-T}{\bar{T}}+3 \beta X^{2} .
$$


If we define a function $\psi(x, t)$ by $\phi(\psi(x, t), t)=x$, we obtain

$$
\psi_{x}=1 / \phi_{X}, \quad \psi_{t}=-\phi_{T} / \phi_{X}
$$

and the equation $\phi_{T}=u_{0}(X)$ is equivalent to $\psi_{t}+u \psi_{x}=0$. In other words, $\psi$ is a singular phase function. Hence we can view $\phi_{T}=u_{0}(X)$ as the eikonal equation "turned inside-out": the miracle is that $\phi$ is smooth, while $\psi$ is not. We extend now this way of looking at things to first order systems.

\section{A.2. Geometric blowup in one space dimension}

\subsection{Some basic definitions}

Consider a system

$$
\partial_{t} u+A(u) \partial_{x} u=0, \quad u \in \mathbb{R}^{N},
$$

where $A(u)$ has eigenvalues $\mu_{1}(u)<\ldots<\mu_{N}(u)$, with corresponding left and right eigenvectors $\ell_{j}(u), r_{k}(u):{ }^{t} \ell_{j} r_{k}=\delta_{j k}$. Just as we did at the end of A.1, we can introduce new coordinates $(X, T)$ and set

$$
u(\phi(X, T), T)=v(X, T)
$$

for some functions $\phi$ and $v$ to be determined. This change of variables yields the new system

$$
v_{T}+\left(A(v)-\phi_{T}\right) \frac{v_{X}}{\phi_{X}}=0
$$

If, for some $k$, we impose

$$
\phi_{T}=\mu_{k}(v),
$$

then, multiplying (2.3) to the left by ${ }^{t} \ell_{j}(v)$, we obtain the equivalent system

$$
{ }^{t} \ell_{k}(v) v_{T}=0, \quad{ }^{t} \ell_{j}(v)\left[\left(\mu_{j}(v)-\mu_{k}(v)\right) v_{X}+\phi_{X} v_{T}\right]=0, \quad j \neq k .
$$

The system (2.4) is called the blowup system of (2.1) (for the eigenvalue $\mu_{k}$ ): observe that its size is $N+1$, and that it is not quasilinear anymore in general. For instance, if we start from Burgers'equation, we obtain the (linear) blowup system

$$
\phi_{T}=v, \quad v_{T}=0,
$$

which is another way of expressing the method of characteristics. It is interesting to compare this with a "partial hodograph" approach which would be to take $u$ as a new space variable. This means that we define $h(X, t)$ by $u(h(X, t), t)=X$, and we have the new equation on $h$

$$
h_{t}=X \text {. }
$$


Comparing with (2.2), this hodograph trick would mean choosing a priori $v$ to be $X$, which is less flexible than leaving $v$ free.

If we start from a $2 \times 2$ system in diagonal form (that is, the coordinates $u_{1}$ and $u_{2}$ are Riemann invariants), we obtain for its blowup system (if, say, $k=1$ )

$$
\phi_{T}=\mu_{1}(v), \quad \partial_{T} v_{1}=0, \quad\left(\mu_{2}-\mu_{1}\right)(v) \partial_{X} v_{2}+\phi_{X} \partial_{T} v_{2}=0
$$

Suppose now that there exists, near some point $M_{0}$, a smooth solution $(\phi, v)$ of the blowup system such that

$$
\phi_{X}\left(M_{0}\right)=0, \quad v_{X}\left(M_{0}\right) \neq 0 .
$$

Set $\Phi\left(M_{0}\right)=m_{0}$. If there is a function $u$ defined and smooth in some open domain $D$ with $m_{0} \in \bar{D}$ satisfying (2.2), such a $u$ will blowup at $m_{0}$ since $u_{x}(\Phi)=v_{X} / \phi_{X}$.

We call a function like $u$ a blowup solution of the system, and we say that the solution $u$ displays geometric blowup, because the blowup of $u$ does not come from the blowup of $v$, but from the singularity of the change of variables $\Phi$.

Going back to (2.5), we see that

$$
\partial_{x} u_{1}(\Phi)=\partial_{X} v_{1} / \phi_{X}, \quad \partial_{x} u_{2}(\Phi)=-\partial_{T} v_{2} /\left(\mu_{2}-\mu_{1}\right) .
$$

In other words, solutions of the blowup system corresponding to $\mu_{1}$ yield solutions of the system for which only $\partial_{x} u_{1}$ blows up. Of course, taking for the system two uncoupled Burgers'equations, one could easily arrange that both $\partial_{x} u_{1}$ and $\partial_{x} u_{2}$ blowup at the same point. However, we believe that this is an exceptional situation; we believe that, in general, only one $\partial_{x} u_{i}$ blows up, and does not induce the other component in blowing up also. To see this, let us recall the classical computation

$$
D_{1} \tilde{q}_{1}+\left(\partial_{1} \mu_{1}\right)\left(\exp -H_{1}\right) \tilde{q}_{1}^{2}=0, \quad D_{2} \tilde{q}_{2}+\left(\partial_{2} \mu_{2}\right)\left(\exp -H_{2}\right) \tilde{q}_{2}^{2}=0,
$$

where $D_{i}=\partial_{t}+\mu_{i} \partial_{x}$, and the functions $H_{j}(u)$ satisfy

$$
\partial_{2} H_{1}=\left(\partial_{2} \mu_{1}\right) /\left(\mu_{1}-\mu_{2}\right), \quad \partial_{1} H_{2}=-\left(\partial_{1} \mu_{2}\right) /\left(\mu_{1}-\mu_{2}\right) .
$$

Another interesting feature is the following: we have seen that, close to $m_{0}$, the blowup of $\partial_{x} u$ corresponds exactly to the vanishing of $\phi_{X}$. But, from (2.5) we get

$$
\partial_{T}\left(\phi_{X}\right)=\left(\partial_{1} \mu_{1}\right) \partial_{X} v_{1}-\left(\frac{\partial_{2} \mu_{1}}{\left(\mu_{2}-\mu_{1}\right)} \partial_{T} v_{2}\right) \phi_{X}
$$

If $\partial_{1} \mu_{1}$ were identically zero (that is if $\mu_{1}$ were linearly degenerate), $\phi_{X}$, being zero at $m_{0}$, would be zero on the vertical line through $m_{0}$ (which corresponds to the 1 characteristic), and $\partial_{x} u_{1}$ would blowup along this 1-characteristic. In other words, the propagation of the singularities of $\partial_{x} u_{1}$ along the characterisitc correspond to the propagation of zeros of $\phi_{X}$ for the differential equation above. What prevents this propagation is the genuine nonlinearity condition $\partial_{1} \mu_{1} \neq 0$.

Remark that the point of this approach is that to obtain singular solutions of (2.1), we are now looking for smooth solutions of (2.4). 


\subsection{Simple waves revisited}

Let us recall that a $k$-simple wave for (2.1) is a solution $u(x, t)=U(\psi(x, t))$, where $\psi$ is a scalar real function, and

$$
U^{\prime}=r_{k}(U), \quad \psi_{t}+\mu_{k}(U) \psi_{x}=0
$$

Hence, for all $(x, t)$, the image $u(x, t)$ is contained in a single integral curve of $r_{k}$. When a k-simple wave blows up, only the $r_{k}$-component of $\partial_{x} u$ blows up.

Consider now the blowup system (2.4) of a given system (2.1). A special class of solutions to (2.4) is obtained by taking $v \equiv v(X), v^{\prime}(X)$ parallel to $r_{k}(v)$ and $\phi_{T}=\mu_{k}(v(X))$. The corresponding solutions $u$ are seen to be the simple wave solutions of (2.1): thus the blowup solutions defined in 2.1 are more general than the simple wave solutions which blowup. In fact, we believe that the class of blowup solutions (in the sense of 2.1) is large enough to describe the local behavior of any "generic" solution around its first blowup point. Let us remark, as can be easily checked for simple waves, that for a blowup solution $u$, the singular part

$$
\frac{1}{\phi_{X}} v_{X}\left(1,-\phi_{T}\right)
$$

of $\nabla u$ has rank one, hence only one component of $u_{x}$ or $u_{t}$ is unbounded; similarly, the only bad direction is the characteristic direction $\left(\xi=1, \tau=-\mu_{k}(u)\right)$.

To finish this section, let us discuss briefly the issue of stability. Suppose we have a simple wave with an initial datum $u_{0}$, blowing up at $m_{0}$. If we perturb a little $u_{0}$, the new solution will not be a simple wave again, but a blowup solution for the same eigenvalue. Conversely, if we perturb a little a blowup solution for $\mu_{k}$, we will obtain again a blowup solution blowing up at a nearby point, for the same $\mu_{k}$. This has been proved for general symmetric systems in any number of space dimensions, see [8].

\section{A.3. Geometric blowup in multidimensional situations (sys- tems)}

\subsection{Blowup systems}

\section{1.a. General definitions}

Consider a quasilinear system (not necessarily symmetric at this stage)

$$
L(u) \equiv \sum_{1 \leq j \leq n} A_{j}(x, u) \partial_{j} u+B(x, u)=0, \quad x \in \mathbb{R}^{n}, \quad u \in \mathbb{R}^{N},
$$

where $A$ and $B$ are real and smooth. Set

$$
A=A(x, u, \xi)=\sum A_{j}(x, u) \xi_{j}
$$

the (matrix) principal symbol of the linearized system of $L$. We make the following Assumption : At some point $\left(x^{0}, u^{0}, \xi^{0}=\left(\xi_{1}^{0}, \ldots, \xi_{n}^{0}\right)\right)$ (with $\xi_{1}^{0}>0$ ), the matrix $A\left(x^{0}, u^{0}, \xi^{0}\right)$ has zero as a simple eigenvalue. 
For $(x, u, \xi)$ close to $\left(x^{0}, u^{0}, \xi^{0}\right)$, we denote by $\lambda(x, u, \xi), r(x, u, \xi), \ell(x, u, \xi)$ the eigenvalue of $A(x, u, \xi)$ continuing zero $\left(\lambda\left(x^{0}, u^{0}, \xi^{0}\right)=0\right)$ and the corresponding right and left eigenvectors. We assume moreover that

$$
\partial_{\xi} \lambda\left(x^{0}, u^{0}, \xi^{0}\right) \neq 0
$$

For some unknown function $\phi(X)$ of the new variables $X=\left(X_{1}, \ldots, X_{n}\right)$, set

$$
\Phi(X)=\left(\phi(X), X_{2}, \ldots, X_{n}\right), \quad \eta(X)=\left(1,-\partial_{2} \phi(X), \ldots,-\partial_{n} \phi(X)\right) .
$$

Definition. The blowup system of (3.1) (corresponding to the eigenvalue $\lambda$ and the coordinate $\left.x_{1}\right)$ is the $(N+1) \times(N+1)$ system in $\phi, v \in R^{N}$

$$
\begin{gathered}
\mathcal{E} \equiv \lambda(\Phi(X), v(X), \eta(X))=0 \\
\mathcal{R}_{1} \equiv{ }^{t} \ell(\Phi, v, \eta) E=0 \\
\mathcal{R}_{j} \equiv{ }^{t} \ell_{j}\left[A(\Phi, v, \eta) \partial_{1} v+\left(\partial_{1} \phi\right) E\right]=0, \quad 2 \leq j \leq N .
\end{gathered}
$$

Here,

$$
E \equiv \sum_{j \geq 2} A_{j}(\Phi, v) \partial_{j} v+B(\Phi, v)
$$

and the $\ell_{j}, j \geq 2$ are any vectors such that $\ell, \ell_{2}, \ldots, \ell_{N}$ is a basis of $R^{N}$. The first equation (3.3a) is called the eikonal equation, for reasons which will become clear later on. In order for the system (3.3) to make sense, it is understood that we consider only smooth solutions $(\phi, v)$ near a point $M_{0}$, satisfying

$$
\Phi\left(M_{0}\right)=x^{0}, \quad v\left(M_{0}\right)=u^{0}, \quad \eta\left(M_{0}\right)=\mu \xi^{0}, \quad \mu>0 .
$$

It is important to note that at a point where $\phi_{X}=0, \partial_{1} v$ will be colinear to $r$.

We have already seen in 2.1 some examples of blowup systems (with respect to $x$ ) in the plane $(x, t)$. Let us consider now, in $\mathbb{R}^{3}$ with coordinates $(x, y, t)$, the scalar equation

$$
\partial_{x t}^{2} u+\left(\partial_{x} u\right)\left(\partial_{x}^{2} u\right)+a \partial_{y}^{2} u=0
$$

Setting $u_{1}=\partial_{x} u, u_{2}=\partial_{y} u$, we obtain the equivalent system

$$
\partial_{t} u_{1}+u_{1} \partial_{x} u_{1}+a \partial_{y} u_{2}=0, \quad \partial_{x} u_{2}-\partial_{y} u_{1}=0,
$$

we can be written

$$
A_{1} \partial_{x} U+A_{2} \partial_{y} U+A_{3} \partial_{t} U=0
$$

with

$$
A_{1}=\left(\begin{array}{cc}
u_{1} & 0 \\
0 & 1
\end{array}\right), \quad A_{2}=\left(\begin{array}{cc}
0 & a \\
-1 & 0
\end{array}\right), \quad A_{3}=\left(\begin{array}{ll}
1 & 0 \\
0 & 0
\end{array}\right)
$$


The determinant of $A=A_{1}-\phi_{y} A_{2}-\phi_{t} A_{3}$ is $\partial_{1} v-\phi_{t}+a \phi_{y}^{2}$. Hence the eikonal equation is

$$
\phi_{t}=\partial_{1} v+a \phi_{y}^{2}
$$

The left eigenvectors corresponding to the eigenvalues $\mu_{1}=0$ and $\mu_{2}=1+\partial_{1} v-\phi_{t}$ of $A$ are ${ }^{t} \ell_{1}=\left(1, a \phi_{y}\right)$ and ${ }^{t} \ell_{2}=\left(\phi_{y}, 1\right)$. Multiplying the system to the left by $\ell_{i}$ yields

$$
\begin{gathered}
\mathcal{R}_{1}=\partial_{T} v_{1}+a \partial_{Y} v_{2}-a \phi_{Y} \partial_{Y} v_{1}=0 \\
\mathcal{R}_{2}=\mu_{2}\left(\phi_{Y} \partial_{1} v_{1}+\partial_{1} v_{2}\right)+\left(\phi_{X}\right)\left(-\partial_{Y} v_{1}+a \phi_{Y} \partial_{Y} V_{2}+\phi_{Y} \partial_{T} v_{1}\right)=0
\end{gathered}
$$

which simplifies to give the blowup system of (3.6)

$$
\begin{gathered}
\phi_{T}=v_{1}+a \phi_{Y}^{2} \\
\partial_{T} v_{1}-a \phi_{Y} \partial_{Y} v_{1}+a \partial_{Y} v_{2}=0 \\
\partial_{X} v_{2}+\phi_{Y} \partial_{X} v_{1}-\phi_{X} \partial_{Y} v_{1}=0
\end{gathered}
$$

At this stage, one has the feeling that all the geometry of the original system is lost. This is not true, because, as we shall see, the geometry never gets lost! For instance, let us compute

$$
\partial_{1} \mathcal{E}=\left(\partial_{1} \phi\right) \partial_{x_{1}} \lambda+\left(\partial_{u} \lambda\right)\left(\partial_{1} v\right)-\sum_{j \geq 2}\left(\partial_{\xi_{j}} \lambda\right) \partial_{j}\left(\partial_{1} \phi\right)=0
$$

We see that, generalizing the remarks from 2.1, what prevents the zeroes of $\phi_{X}$ from propagating is the non vanishing of $\left(\partial_{u} \lambda\right)\left(\partial_{1} v\right)$. If $\partial_{1} v \neq 0$, this is equivalent to the non vanishing of $r \partial_{u} \lambda$, that is, to the (microlocal) genuine non linearity condition of Lax.

\section{1.b. Some explanations about the choice of the blowup system}

If we perform in (3.1) the change $u(\Phi)=v$, the system becomes

$$
\left(A_{1}-\sum_{j \geq 2} A_{j} \partial_{j} \phi\right) \frac{\partial_{1} v}{\partial_{1} \phi}+E=0 .
$$

Getting an actual blowup solution $u$ requires that $\partial_{1} v$ should not be proportional to $\partial_{1} \phi$, hence we require the matrix

$$
A_{1}-\sum_{j \geq 2} A_{j} \partial_{j} \phi
$$

to be noninvertible, which gives the eikonal equation. Multiplying finally (3.8) to the left by ${ }^{t} \ell,{ }^{t} \ell_{j}$ yields $(3.3 \mathrm{~b}),(3.3 \mathrm{c})$.

There is of course some arbitrariness in the choice of $\Phi$ and $v$ satisfying $u(\Phi)=v$, since we can replace $\Phi$ by $\Phi\left(\Phi_{1}\right)$ and $v$ by $v\left(\Phi_{1}\right)$ for an arbitrary diffeomorphism $\Phi_{1}$. What we are really looking for here is a mapping $\Phi$ for which $\Phi^{\prime}$ is not invertible at some point $M_{0}$, but such that $\Phi^{\prime}\left(M_{0}\right)$ has one dimensional kernel. The choice (3.2) is then no restriction, and allows simple formulations. 


\subsection{Blowup solutions, folds and cusps}

\section{2.a. Definitions}

We first give a precise meaning to $u(\Phi)=v$.

Definition. Let be given a smooth solution $(\phi, v)$ of the blowup system (3.3). Suppose that there exists an open domain $D\left(x^{0} \in \bar{D}\right)$ and a mapping $\psi \in C^{0}(\bar{D})$ with values in $R^{n}$ verifying

$$
\psi\left(x^{0}\right)=M_{0}, \quad \Phi(\psi(x))=x, \quad \operatorname{det} \Phi^{\prime}(\psi(x)) \neq 0, \quad x \in D .
$$

Then the function $u(x)=v(\psi(x))$ is a smooth solution of $L(u)=0$ in $D$, continuous in $\bar{D}$. If in addition

$$
\partial_{1} \phi\left(M_{0}\right)=0, \quad \partial_{1} v\left(M_{0}\right) \neq 0,
$$

then $\nabla u$ blows up at $x^{0}$. Such a solution $u$ is called a (geometric) blowup solution.

Note that to the same solution $(\phi, v)$ one can associate several solutions $u$, depending on the choices of $D$ and $\psi$ (see below the basic examples). The set of the blowup solutions depends on the eigenvalue chosen to blowup the original system, but not on the coordinate $x_{1}$ (one can take instead any $x_{j}$ provided $\xi_{j}^{0}>0$ ). In general, the following theorem describes the way $u^{\prime}$ blows up at $x^{0}$.

Theoreme. Let $(\phi, v)$ be a smooth solution of the blowup system for which

$$
\partial_{1} \phi\left(M_{0}\right)=0, \quad \partial_{1} v\left(M_{0}\right) \neq 0,
$$

and let $(u, \psi, D)$ an associated blowup solution. Set $\xi(x)=\eta(\psi(x))$. Then, for $x \in D$,

$$
u^{\prime}(x)=C(x)\left[\partial_{1} \phi(\psi(x))\right]^{-1} r(x, u(x), \xi(x))^{t} \xi(x)+R(x),
$$

where $C$ and $R$ are continuous on $\bar{D}$ and $C\left(x^{0}\right) \neq 0$.

This theorem generalizes what has been emphasized at the end of 2.2: only one component of $u^{\prime}$ blows up, and there is only one bad direction, namely, $\xi(x)$. From this theorem, we see that the analysis of the blowup of $u^{\prime}$ for a blowup solution is a purely geometrical local problem at $M_{0}$ about the behavior of $\Phi$ and $\psi$.

The two simplest special cases are the following.

Definition. Assume that, for a smooth solution $(\phi, v)$ of the blowup system,

$$
\partial_{1} \phi\left(M_{0}\right)=0, \quad \partial_{1}^{2} \phi\left(M_{0}\right) \neq 0,
$$

that is, $\Phi$ has a fold singularity at $M_{0}$. We call then any blowup solution associated with $(\phi, v)$ a fold solution.

Assume that, for a smooth soluion $(\phi, v)$ of the blowup system,

$$
\partial_{1} \phi\left(M_{0}\right)=0, \quad \partial_{1}^{2} \phi\left(M_{0}\right)=0, \quad \partial_{1}^{3} \phi\left(M_{0}\right) \neq 0, \quad d\left(\partial_{1} \phi\right)\left(M_{0}\right) \neq 0,
$$

that is, $\Phi$ has a cusp singularity at $M_{0}$. We call then any blowup solution associated with $(\phi, v)$ a cusp solution. 
Define now $S_{1}$ to be $\left\{X, \partial_{1} \phi(X)=0\right\}$, and $S=\Phi\left(S_{1}\right)$.

In the fold case, $S$ is a characteristic surface (with normal $\xi(x)$ ), and the image of $\Phi$ is one of the closed "half-spaces" limited by $S$ : if we take $D$ to be some open neighbourhood of $x^{0}$ in this half-space, there are two possible choices for $\psi$, and in either case one can show that

$$
C^{-1} d^{1 / 2} \leq\left|\partial_{1} \phi(\psi(x))\right| \leq C d^{1 / 2}
$$

for some $C>0, d$ being the distance to $S$.

This has an important consequence. Suppose now we are considering a hyperbolic system or equation with respect to $t$, and look for a first blowup point $m$ (that is, a blowup point $m=(x, T)$ where $T$ is the lifespan of the solution). If the solution close to $m$ is a blowup solution in the sense above, it cannot be a fold solution, since the corresponding characteristic surface through $m$ cannot be contained in $\{t \geq T\}$. We suggest the following conjecture:

Conjecture (belief ?). Generically, the solution of a stricly hyperbolic system close to a first blowup point is a blowup solution of cusp type for one eigenvalue of the system.

Up to now, we have only considered blowup solutions corresponding to maps $\Phi$ with $\Phi^{\prime}\left(M_{0}\right)$ having a one dimensional kernel. As emphasized in the above theorem, these solutions behave, asymptotically near $\Phi\left(M_{0}\right)$, pretty much like one dimensional simple waves. It is also possible to construct solutions which display the behavior of a superposition of two blowup solutions, corresponding to two different directions $\xi_{1}$ and $\xi_{2}$. Such solutions exist only in truely multidimensional situations. This has been achieved for quasilinear wave equations in [6].

\section{2.b. Existence in the analytic case}

In view of the above definition of a blowup solution, it is important to show that it is actually possible to find smooth solutions of the blowup system for which

$$
\partial_{1} \phi\left(M_{0}\right)=0, \quad \partial_{1} v\left(M_{0}\right) \neq 0 .
$$

We only want here to make sure that no (hidden) degeneracy of the blowup system forces $\partial_{1} v$ to vanish when $\partial_{1} \phi$ does. It is enough for this to consider here the analytic case where $A_{j}$ and $B$ are assumed to be analytic of their arguments. Without stating a precise theorem here (see [1]), let us state roughly the following: by choosing appropriately Cauchy data and using the Cauchy-Kovalesvski theorem, one can contruct

i) Fold solutions in all dimensions,

ii) Cusp solutions if $n \geq 3$,

iii) Cusp solutions for $n=2$ if

$$
\left(r \partial_{u} \lambda\right)\left(x^{0}, u^{0}, \xi^{0}\right) \neq 0 .
$$


Let us comment a little about this: the definitions in 3.2 have been given in general, and apply as well to a linear system; in this case, fold solutions will be singular along a characteristic surface as in the general case, and cusp solutions will correspond to a "propagated" plane cusp. It is not possible to obtain a cusp solution in the plane for a linear system: the system has to be sufficiently nonlinear, and condition (3.9) expressing the "genuine nonlinearity" of $\lambda$ (in the sense of Lax) is the relevant condition.

\section{A.4. Geometric blowup in multidimensional situations (scalar second order equations)}

We have given above the definitions and basic properties of blowup solutions for a first order quasilinear system, since the geometry is more transparent in this case. In practice, one often considers second order scalar equations. Though such equations can be in principle written as first order systems (as we did for (3.5)), this leads to oversized systems in higher dimensions, and it is better to stick to the scalar character of the original equation. We give below an alternative approach to the construction of the blowup system in this case. Let us consider a second order equation

$$
P(u) \equiv \sum p_{i j}(x, u, \nabla u) \partial_{i j}^{2} u+q(x, u, \nabla u)=0 .
$$

We set $x=\left(x_{1}, x^{\prime}\right), x^{\prime}=\left(x_{2}, \ldots, x_{n}\right)$. For new variables $s \in R, y=\left(y_{2}, \ldots, y_{n}\right)$, and a function $\phi(s, y)$, we define

$$
\begin{gathered}
\bar{\partial}=\left(0, \partial_{y}\right), \quad \hat{\phi}=\left(-1, \phi_{y}\right), \\
\Phi(s, y)=(\phi(s, y), y), \quad w=u(\Phi), \quad v=\left(\partial_{1} u\right)(\Phi) .
\end{gathered}
$$

From the very definition, we note that

$$
\mathcal{A} \equiv w_{s}-\phi_{s} v=0
$$

and we call this the auxiliary equation.

Proposition. With the above notations, we have

$$
P(u)(\Phi)=\mathcal{E} \frac{v_{s}}{\phi_{s}}+\mathcal{R}
$$

where

$$
\begin{gathered}
\mathcal{E} \equiv \sum p_{i j}(\phi, y, w, \bar{\partial} w-\hat{\phi} v) \hat{\phi}_{i} \hat{\phi}_{j} \\
\mathcal{R} \equiv \sum p_{i j}(\phi, y, w, \bar{\partial} w-\hat{\phi} v)\left[\bar{\partial}_{i j}^{2} w-v \bar{\partial}_{i j}^{2} \phi-\left(\hat{\phi}_{i} \bar{\partial}_{j}+\hat{\phi}_{j} \bar{\partial}_{i}\right) v\right]+q(\phi, y, w, \bar{\partial} w-\hat{\phi} v) .
\end{gathered}
$$

This Proposition leads us to define the blowup system of (4.1) as the $3 \times 3$ system in the unknown $(\phi, v, w)$

$$
\mathcal{A}=0, \quad \mathcal{E}=0, \quad \mathcal{R}=0,
$$


the second and third equations being respectively the eikonal equation and the residual equation .

Just as above, a blowup solution $u$ will correspond to a smooth solution $(\phi, v, w)$ of the blowup system for which $\phi_{s}\left(M_{0}\right)=0, v_{s}\left(M_{0}\right) \neq 0$. Remark that $u$ and $\nabla u$ are continuous in this case, only $\nabla^{2} u$ blows up at $x^{0}$ : in fact, $\partial_{1}^{2} u(\Phi)=v_{s} / \phi_{s}$.

If we apply this procedure to the simple equation

$$
u_{t t}-\partial_{x}\left(p\left(u_{x}\right)\right)=0
$$

we obtain

$$
\mathcal{E}=\phi_{t}^{2}-p^{\prime}(v), \quad \mathcal{R}=w_{t t}-2 v_{t} \phi_{t}-v \phi_{t t}=0 .
$$

If we choose for instance $\phi_{t}=c(v)$, we remark that $\mathcal{R}=\partial_{t}\left(w_{t}-c v-C(v)\right)$, where $C^{\prime}=c$, hence we can integrate and write

$$
w_{t}-c v-C=f(s)
$$

It is easy to check that this is just what can be obtained from writing the equation as a $p$-system, writing the system using the Riemann invariants $u_{t} \pm C\left(u_{x}\right)$, and straightening out the second characteristics!

If we apply this to the example (3.5), we find (with self-explaining notations)

$$
\mathcal{E}=-\phi_{T}+v+a \phi_{Y}^{2}, \quad \mathcal{R}=v_{T}+a\left(\partial_{Y}^{2} w-v \partial_{Y}^{2} \phi-2 \phi_{Y} v_{Y}\right)
$$

Note that $\mathcal{R}_{X}$ is obtained by eliminating $v_{2}$ from the blowup system (3.7).

Remark that all singular solutions we have constructed so far (including that of [6]) blow up like $C(T-t)^{-1}$. This seems to be rather general for equations or systems with genuinely nonlinear simple eigenvalues. There are many unanswered questions about what the constants $C$ can be. An introduction to this problem and some results can be found in [7].

\section{B. STRUCTURE OF THE LINEARIZED BLOWUP SYSTEMS}

We limit ourselves here to the case of scalar equations, which is simpler. The corresponding theorems for general systems can be found in [8]. We would like to emphasize here that the blowup process explained in $\mathbf{A}$ is a transformation at the level of the nonlinear system: from a nonlinear system, it produces a new nonlinear system. The linearization takes place on this new system: linearizing the original system on an approximate singular solution would a disaster. This can be seen for instance in the following way: suppose we have a blowup solution $\bar{u}$ of Burgers'equation, with initial data $\bar{u}_{0}$. If we perturb this initial value and look for the corresponding solution $u$ as $u=\bar{u}+\dot{u}$, one can compute an approximation of $u$ by linearizing on $\bar{u}$. This leads to a very bad understanding of the new blowup time for $u$. 


\section{B.1. The linearized blowup system of a scalar second order equation}

In A.4, we discussed the equation

$$
P(u) \equiv \sum p_{i j}(x, u, \nabla u) \partial_{i j}^{2} u+q(x, u, \nabla u)=0,
$$

and its blowup system

$$
\mathcal{A}=0, \quad \mathcal{E}=0, \quad \mathcal{R}=0
$$

We denote by

$$
\mathcal{E}_{\phi, v, w}^{\prime}(\dot{\phi}, \dot{v}, \dot{w}) \equiv \mathcal{E}^{\prime}(\dot{\phi}, \dot{v}, \dot{w}) \equiv \mathcal{E}^{\prime}
$$

the differential of $\mathcal{E}$ at the point $(\phi, v, w)$, and similarly for $\mathcal{A}$ and $\mathcal{R}$. Let us define now, with the notations of A.4,

$$
\begin{gathered}
\gamma=\left(\sum \partial_{\nabla u} p_{i j} \hat{\phi}_{i} \hat{\phi}_{j}\right) \hat{\phi} \\
Z_{1}=\sum p_{i j}\left(\hat{\phi}_{i} \bar{\partial}_{j}+\hat{\phi}_{j} \bar{\partial}_{i}\right), \\
Q=\sum p_{i j} \bar{\partial}_{i j}^{2},
\end{gathered}
$$

where as usual $p_{i j}=p_{i j}(\phi, y, w, \bar{\partial} w-\hat{\phi} v)$. If we call tangential the $y$-variables, we see that $Z_{1}$ is a tangential field, and $Q$ is the tangential part of the (principal part of the) linearized operator of $P$.

The following straightforward proposition uses these notations to describe the linearized blowup system.

Proposition. Set $\dot{z}=\dot{w}-v \dot{\phi}$. Then

$$
\begin{gathered}
\mathcal{E}^{\prime}(\dot{\phi}, \dot{v}, \dot{w})=-\gamma \dot{v}+Z_{1} \dot{\phi}+Z_{2} \dot{z}+a_{0} \dot{\phi}+b_{0} \dot{z}, \\
\mathcal{R}^{\prime}(\dot{\phi}, \dot{v}, \dot{w})=Q \dot{z}-Z_{1} \dot{v}+c_{1} \bar{\partial} \dot{z}+c_{0} \dot{z}+c_{2} \dot{\phi}+c_{3} \dot{v} \\
\mathcal{A}^{\prime}(\dot{\phi}, \dot{v}, \dot{w})=\dot{z}_{s}+v_{s} \dot{\phi}-\phi_{s} \dot{v}
\end{gathered}
$$

where $Z_{2}$ is a tangential field and $a_{0}, b_{0}, c_{0}, c_{1}, c_{2}, c_{3}$ are smooth coefficients depending on $(\phi, v, w)$, which we need not know explicitly here.

For instance, in the basic example A (3.5)

$$
P(u)=\partial_{x t}^{2} u+\left(\partial_{x} u\right)\left(\partial_{x}^{2} u\right)+a \partial_{y}^{2} u=0
$$

we obtain

$$
\begin{gathered}
\gamma=-1, \quad Z_{1}=-\left(\partial_{T}-2 a \phi_{Y} \partial_{Y}\right), \quad Q=a \partial_{Y}^{2} \\
\mathcal{E}^{\prime}=Z_{1} \dot{\phi}+\dot{v}, \quad \mathcal{R}^{\prime}=Q \dot{z}-Z_{1} \dot{v}+(Q v) \dot{\phi}
\end{gathered}
$$




\section{B.2. Geometric interpretation}

i) We explain first the introduction of $\dot{z}$ instead of $\dot{w}$ : when we linearize the relation $u(\Phi)=w$, we get $\dot{u}(\Phi)+u^{\prime}(\Phi) \dot{\Phi}=\dot{w}$. The "physical" object is $u$, not $w$ which depends on the choice of $\Phi$; hence the "physical" variable to consider is $\dot{u}(\Phi)$, not $\dot{w}$. Here, we have, because of the choice of $\Phi$,

$$
\dot{u}(\Phi)=\dot{w}-v \dot{\phi}
$$

which is just $\dot{z}$. We can say that the introduction of $\dot{z}$ cancels the arbitrariness in the choices of $w$ and $\Phi$ discussed in A.3.1.

ii) We interpret now the eikonal equation $\mathcal{E}=0$ : if we set formally

$$
\Phi^{-1}(x)=\left(\psi(x), x^{\prime}\right)
$$

we obtain

$$
(\nabla \psi)(\Phi)=-\frac{1}{\phi_{s}} \hat{\phi}
$$

Hence the eikonal equation is equivalent to

$$
\sum p_{i j}(x, u, \nabla u)\left(\partial_{i} \psi\right)\left(\partial_{j} \psi\right)=0,
$$

that is, the (singular) lagrangean manifold $\Lambda=\{(x, \nabla \psi)\}$ is characteristic for the linearized equation of (1.1); more simply, $\psi$ is a singular phase function.

iii) The field $Z_{1}$ will play a crucial role in the sequence. To understand its geometrical meaning, let us return to the original "physical" coordinates $x$ by computing $\Phi^{\prime} Z_{1}$. We find

$$
\Phi^{\prime} Z_{1}=-\phi_{s} \pi_{*} H_{p}, \quad p=\sum p_{i j}(x, u, \nabla u) \xi_{i} \xi_{j}
$$

where $H_{p}$ is the Hamiltonian field of the principal symbol $p$ of the linearized equation on $u$, taken on $\Lambda$, and $\pi_{*} H_{p}$ is its projection as a field on the space $R_{x}^{n}$. In other words, $\Phi^{\prime} Z_{1}$ is (apart from the factor $-\phi_{s}$ ) the transport equation corresponding to $\Lambda$ (or $\psi)$.

\section{B.3. Decoupling of the linearized blowup system}

We introduce first a definition.

Definition. Let $(\phi, v, w)$ be smooth functions in the domain D. We say that we are in the genuinely nonlinear case if the function

$$
\gamma=\sum \partial_{\partial_{k} u} p_{i j}(\phi, y, w, \bar{\partial} w-\hat{\phi} v) \hat{\phi}_{i} \hat{\phi}_{j} \hat{\phi}_{k}
$$

defined in (1.2) does not vanish in D. 
For instance, in the simplest case of the equation

$$
u_{t t}-\partial_{x}\left(p\left(u_{x}\right)\right)=0,
$$

we get $\gamma=p^{\prime \prime}(v)$.

The remarkable fact is that in a genuinely nonlinear situation, the linearized blowup system almost decouples, as indicated in the following theorem.

Theoreme. In the genuinely nonlinear case, we have, for smooth coefficients $\alpha_{j}$, $1 \leq j \leq 5$ and $\beta_{1}, \beta_{2}$ depending on $(\phi, v, w)$, the identities

$$
\begin{gathered}
\left(Z_{1} \partial_{s}-\phi_{s} Q+Q_{1}\right) \dot{z}+\alpha_{1} Z_{1} \dot{\phi}+\alpha_{2} \dot{\phi}=-\phi_{s} \mathcal{R}^{\prime}+\left(Z_{1}+\alpha_{3}\right) \mathcal{A}^{\prime}+\alpha_{4} \mathcal{E}^{\prime} \\
\left(Z_{1}^{2}+\beta_{1} Z_{1}+\beta_{2}\right) \dot{\phi}+\bar{Q}_{2} \dot{z}=\left(Z_{1}+\alpha_{5}\right) \mathcal{E}^{\prime}-\gamma \mathcal{R}^{\prime} .
\end{gathered}
$$

Here, $Q_{1}$ is a first order operator, and $\bar{Q}_{2}$ a tangential second order operator. Moreover, the coupling coefficients $\alpha_{1}, \alpha_{2}$ are linear combinations of derivatives of $\mathcal{E}(\phi, v, w), \mathcal{R}(\phi, v, w)$, and $\mathcal{A}(\phi, v, w)$.

The point of this theorem is that, when solving the blowup system by an iteration process, we linearize on $(\phi, v, w)$ for which $\mathcal{E}, \mathcal{R}, \mathcal{A}$ are already very small; hence the coupling coefficients $\alpha_{1}, \alpha_{2}$ (which prevent the system (3.1) (3.2) from being triangular) are also very small.

Rather than give general formulas which can be found in [5], let us display the exact form of the theorem in the special case of our model operator

$$
\partial_{x t}^{2} u+\left(\partial_{x} u\right)\left(\partial_{x}^{2} u\right)+a \partial_{y}^{2} u=0
$$

We find

$$
\begin{array}{r}
Z_{1} \partial_{s} \dot{z}-a \phi_{s} \partial_{Y}^{2} \dot{z}+\left(a \partial_{Y}^{2} \phi\right) \partial_{s} \dot{z}+\alpha_{1} Z_{1} \dot{\phi}+\alpha_{2} \dot{\phi}=-\phi_{s} \mathcal{R}^{\prime}+\left(Z_{1}-a \partial_{Y}^{2} \phi\right) \mathcal{A}^{\prime}+Z_{1} \phi_{s} \mathcal{E}^{\prime}, \\
Z_{1}^{2} \dot{\phi}+\left(a \partial_{Y}^{2} \phi\right) Z_{1} \dot{\phi}+\left(a \partial_{Y}^{2} v\right) \dot{\phi}+a \partial_{Y}^{2} \dot{z}=\left(Z_{1}+a \partial_{Y}^{2} \phi\right) \mathcal{E}^{\prime}+\mathcal{R}^{\prime}
\end{array}
$$

Here,

$$
\alpha_{1}=\partial_{s} \mathcal{E}, \quad \alpha_{2}=a \partial_{Y}^{2} \mathcal{A}-\partial_{s} \mathcal{R}
$$

\section{B.4. How to solve the linearized blowup system}

Suppose that we can solve exactly in $D$ the system $(3.1),(3.2)$ in $(\dot{z}, \dot{\phi})$ with $\mathcal{E}^{\prime}$, $\mathcal{R}^{\prime}$ and $\mathcal{A}^{\prime}$ replaced by given quantities $\dot{f}, \dot{g}$ and $\dot{h}$; determine now $\dot{v}$ from $\mathcal{E}^{\prime}=\dot{f}$, thanks to $(1.5 \mathrm{a})$. For the functions $(\dot{\phi}, \dot{v}, \dot{w})$ thus obtained, we have then

$$
\mathcal{E}^{\prime}=\dot{f}, \quad \mathcal{R}^{\prime}=\dot{g}, \quad\left(Z_{1}+\alpha_{3}\right)\left(\mathcal{A}^{\prime}-\dot{h}\right)=0 .
$$

Taking into account the boundary conditions on $(\phi, v, w)$ (hence on $(\dot{\phi}, \dot{v}, \dot{w}))$, suppose we can ensure that $\mathcal{A}^{\prime}-\dot{h}$ vanishes on some part $\Gamma$ of the boundary of $D$; suppose also that $D$ is under the influence of $\Gamma$ for $Z_{1}$ : then we obtain $\mathcal{A}^{\prime}=\dot{h}$, and 
the linearized system is solved in $D$. We will see in $\mathrm{C}$, in some special case, how and why these conditions can be achieved.

Let us remark finally that we cannot just neglect the coupling terms $\alpha_{1} Z_{1} \dot{\phi}$ and $\alpha_{2} \dot{\phi}$ in (3.1), (3.2), though these are quadratic error terms: this would lead, in the actual solution of the linearized system

$$
\mathcal{E}^{\prime}=\dot{f}, \quad \mathcal{R}^{\prime}=\dot{g}, \quad \mathcal{A}^{\prime}=\dot{h},
$$

to quadratic errors divided by $\phi_{s}$, a singularity which is not admissible for us, because we want to solve in smooth functions.

We have seen in parts A and B the algebraic aspects of the geometric blowup theory: definition of the blowup system, structure of the linearized system. We are going to see now how the blowup of small classical solutions to quasilinear wave equations can be fitted into this framework.

\section{REDUCTION TO A PROBLEM ON A FIXED COMPACT DO- MAIN}

We go back now to one of the situations we have discussed in chapter I: genuinely nonlinear wave equations in dimensions $n=2$ or $n=3$ (that is, cases (1) or (2)); equations satisfying the first null condition in dimension $n=2$ (that is, case (3)). In all three cases, we assume the condition (ND). To prove the blowup of $u$, the idea is to construct a piece of blowup solution $\tilde{u}$ in the form

$$
\tilde{u}=\epsilon r^{-\frac{n-1}{2}} G(r-t, \omega, \tau),
$$

where $G$ is a smooth function of the reduced variables $\sigma=r-t, \omega, \tau$, blowing up for $\tau=\bar{\tau}_{\epsilon}$. Recall that $\bar{\tau}_{\epsilon}$ is precisely the number we are looking for: it is the expression of the lifespan in the slow time variable. As explained in chapter I, it is expected to be closed to $\bar{\tau}$. Recall also that the slow time $\tau$ depends on the particular problem we are considering: for the three problems mentioned above, we have respectively

$$
\tau=\epsilon t^{1 / 2}, \quad \tau=\epsilon \log t, \quad \tau=\epsilon^{2} \log t .
$$

This piece $\tilde{u}$ will be defined in a domain

$$
-C \leq \sigma \leq M, \quad \tau_{0} \leq \tau \leq \bar{\tau}_{\epsilon}, \omega \in S^{n-1} .
$$

Arranging this domain to make it an influence domain, we obtain $u=\tilde{u}$ there, proving that the lifespan has to verify $\tau \leq \bar{\tau}_{\epsilon}$.

We set

$$
u(x, t)=\epsilon r^{-\frac{n-1}{2}} v(x, t)=\epsilon r^{-\frac{n-1}{2}} G(r-t, \omega, \tau) .
$$

For this programm to work, an obvious necessary condition is that $v$ can be considered, in a domain where $\tau_{0} \leq \tau \leq \tau_{1}<\bar{\tau}$, as a smooth function of the reduced variables. 


\section{C.1. The solution $u$ close to the boundary of the light cone}

\subsection{A first approximation of $u$}

In all three cases, the existence of the solution $u$ has been obtained using Klainerman's energy method. More precisely, following [16] (see also [9], [17] or [22] for the third case), the method of proof uses an approximate solution $u_{a}$, and yields a control of $\dot{u}=u-u_{a}$. We will not enter into the details of the constructions of $u_{a}$. All we need here is that, in a strip $-C \leq \sigma \leq M, \tau_{0} \leq \tau \leq \tau_{1}$, we have

$$
u_{a}=\epsilon r^{-\frac{n-1}{2}} G_{0}(\sigma, \omega, \tau),
$$

where $G_{0}$ is the solution of the reduced equation defined in I.6. We thus have in this strip

$$
\left|Z^{\alpha}\left(u-\epsilon r^{-\frac{n-1}{2}} G_{0}\right)\right| \leq o(\epsilon)(1+t)^{-\frac{n-1}{2}} .
$$

If we want a more precise construction for $u_{a}$, it turns out that we only need add to $G_{0}$ above more and more terms which also are smooth functions of the reduced variables. Thus, we can improve estimates like (1.1) to gain any power of $\epsilon$ instead of $o(\epsilon)$ in the right-hand side of (1.1). This will allow us to consider $G$ as a smooth enough function of its variables. This is what we used in [4] and [5] for case (1). For cases $(2)$ or $(3)$, we can use a better device.

\subsection{The function $G$ as a smooth function of its variables}

It turns out that $u$ has in fact an even better behavior than the one indicated in (1.1). It is expressed in term of the special field $X=t\left(\partial_{t}+\partial_{r}\right)$.

Proposition (case (2)). Assume that u satisfies the estimates

$$
\left|Z^{\alpha} u\right| \leq C \epsilon(1+t)^{-\frac{n-1}{2}} .
$$

Then we have

$$
\left|Z^{\alpha} X^{k} v\right| \leq C \epsilon^{k}
$$

Proposition (case (3)). Assume that u satisfies (1.2). Then we have

$$
\left|Z^{\alpha} X^{k} v\right| \leq C \epsilon^{2 k} .
$$

The meaning of these propositions is this: suppose we define $G$ by

$$
G(\sigma, \omega, \tau)=v(x, t) .
$$

Then $G$ has bounded derivatives in its variables. In fact, $v$ is already a smooth function with bounded derivatives in the variables $(r, \omega, t)$. Now

$$
\partial_{\tau}=(d t / d \tau)\left(\partial_{t}+\partial_{r}\right),
$$


and, respectively, $d t / d \tau=t / \epsilon^{2}, d t / d \tau=t / \epsilon$. Since

$$
S+\sum \omega_{i} h_{i}=(r+t)\left(\partial_{t}+\partial_{r}\right)
$$

we can deduce $\left|Z^{\alpha} X^{k} v\right| \leq C$ from (1.2). The stronger information of the propositions is obtained using again the equation on $u$. To do this, we first rewrite the equation using polar coordinates; then we factor the (main) radial part of this equation as

$$
\partial_{t}^{2}+(\ldots) \partial_{r t}^{2}+(\ldots) \partial_{r}^{2}=\left(\partial_{t}-\lambda_{+} \partial_{r}\right)\left(\partial_{t}-\lambda_{-} \partial_{r}\right)+\ldots,
$$

where $\lambda_{ \pm}$are close to \pm 1 . Finally, we rewrite the main part (involving $\partial_{t}, \partial_{r}$ ) using $X$. We obtain respectively

$$
\begin{gathered}
\left(\partial_{t}-\lambda_{+} \partial_{r}\right) X v=t^{-1 / 2} f_{0} X v+t^{-1 / 2} f_{0}+\epsilon^{2} f_{0}, \\
\left(\partial_{t}-\lambda_{+} \partial_{r}\right) X v=t^{-1} X v+t^{-1} f_{0}+\epsilon f_{0} .
\end{gathered}
$$

Here, $f_{0}$ denotes any smooth function of $\epsilon, \sigma, \omega, t^{-\frac{n-1}{2}}, Z^{\beta} v$. This gives the propositions for $k=1$. A more elaborate writing of $\left(\partial_{t}-\lambda_{+} \partial_{r}\right)\left(Z^{\alpha} X^{k} v\right)$ gives the general case.

\section{C.2. The free boundary Goursat problem}

\subsection{The exact equation}

Setting now

$$
u=\epsilon r^{-\frac{n-1}{2}} G(\sigma, \omega, \tau),
$$

we obtain a new equation on $G$, that we want to solve all the way up to the blowup time $\bar{\tau}_{\epsilon}$, knowing already that a solution exists close to $\tau_{0}$. Remember that this equation is, for $\epsilon=0$, the reduced equation obtained in I.6. For $\epsilon>0$, it contains additionnal first and second order derivatives of $G$. Hence here, it is the equation which is perturbed; this is different from the situation of [8], where we perturb the data instead. To obtain such a $G$, we will blowup the full equation on $G$ by setting

$$
\sigma=\phi(s, \omega, \tau)
$$

and keeping the variables $\omega$ and $\tau$. According to the theory of $\mathbf{A}$, we have now to solve the blowup system $\mathcal{L}(\phi, v, w)=0$ for $\phi, v, w$. Of course, the explicit writing of this blowup system is extremely tedious, even in the simplest cases. For instance, for the $2 \mathrm{D}$ model case

$$
\left(1-u_{t}\right) u_{t t}-\Delta u=0
$$

of [4], we obtain the equation

$$
\begin{gathered}
-G_{\sigma \tau}+\frac{\tau}{\left(\tau^{2}+\epsilon^{2} \sigma\right)^{1 / 2}} G_{\sigma} G_{\sigma \sigma}-\epsilon^{2}\left[\frac { \tau } { ( \tau ^ { 2 } + \epsilon ^ { 2 } \sigma ) ^ { 2 } } \left(\frac{G}{4}+G_{\omega \omega}+\frac{G_{\tau} G_{\sigma \sigma}}{2\left(\tau^{2}+\epsilon^{2} \sigma\right)^{1 / 2}}\right.\right. \\
\left.+\frac{G_{\tau}-\tau G_{\tau \tau}}{4 \tau^{2}}+\frac{\tau}{\left(\tau^{2}+\epsilon^{2} \sigma\right)^{1 / 2}}\left(G_{\sigma}-\frac{\epsilon^{2}}{2 \tau} G_{\tau}\right)\left(G_{\sigma \tau}+\frac{\epsilon^{2}}{4 \tau^{3}} G_{\tau}-\frac{\epsilon^{2}}{4 \tau^{2}} G_{\tau \tau}\right)\right]=0
\end{gathered}
$$




\subsection{The stability lemma}

Since we know the structure of the equations on $G$, the algebraic analysis of the linearized blowup system B will tell us enough about its structure to allow us to prove tame estimates. We have seen in chapter $\mathbf{I}$ that, for $\epsilon=0$, the equations on $G$ are essentially Burgers'equations. To understand the linearized blowup system, we primarily have to know the structure of $Z_{1}$ and $Q$. Since for $\epsilon=0$, the linearized blowup system reduces to

$$
\partial_{\tau s}^{2} \dot{z}=\ldots
$$

we have to solve a Goursat problem. More precisely, it is easy to construct an approximate solution of the blowup system, which is exact close to $\left\{\tau=\tau_{0}\right\}$, and flat on $\{s=M\}$ (which corresponds to $\sigma=M$ ). The blowup time of the solution corresponding to this approximation is, of course, $\bar{\tau}$. We are looking for a solution of the blowup system which is a perturbation of this approximate solution: we will solve the linearized system in flat functions on $\left\{\tau=\tau_{0}\right\}$ and $\{s=M\}$.

The main difficulty here is this: ideally, one would like to solve the blowup system in a big domain

$$
-C \leq s \leq M, \quad \tau_{0} \leq \tau \leq \tau_{2}
$$

with $\tau_{2}>\bar{\tau}$. The blowup time $\bar{\tau}_{\epsilon}$ would then be obtained as the lowest value of $\tau$ on the surface $\left\{\phi_{s}=0\right\}$. This is the way one can work in 1D (see [2]), but it does not seem possible in multidimensional situations. In fact, even for the simplified model of the linearized blowup system

$$
\partial_{\tau s}^{2} \dot{z}+\epsilon^{2} \phi_{s} \partial_{\omega}^{2} \dot{z}=\dot{f}
$$

it does not seem possible to obtain energy estimates in a domain where $\phi_{s}$ may take both positive and negative values. Hence we have to stay in the domain $\tau \leq \bar{\tau}_{\epsilon}$. But $\bar{\tau}_{\epsilon}$ is precisely part of the problem: we have a free boundary problem.

To handle this problem, we introduce a parameter $\lambda$ close to zero and perform the change of variables ( $t$ has nothing to do with the old $t$ !)

$$
\tau=\tau_{0}+t+\lambda t(1-\chi(t))
$$

where $\chi(t)$ is one near $t=0$ and zero near $t=t_{0}=\bar{\tau}-\tau_{0}$. We denote by $\tilde{\mathcal{L}}(\lambda, \phi, v, w)=0$ the system obtained from $\mathcal{L}$ by the change $(2.1)$. We have now to solve $\tilde{\mathcal{L}}=0$ in a fixed domain

$$
D=\left\{-C \leq s \leq M, \quad 0 \leq t \leq t_{0}, \quad \omega \in S^{n-1}\right\}
$$

We will arrange for $\phi_{s}$ to vanish only at some point on $\left\{t=t_{0}\right\}$, thus at the end the lifespan will be just

$$
\bar{\tau}_{\epsilon}=\tau_{0}+(1+\lambda) t_{0}
$$


We say that $\phi$ satisfies condition (H) in $D$ if, for some point $M \in \bar{D}$ with $t=t_{0}$, we have

$$
\left\{\begin{array}{l}
\phi_{s} \geq 0, \quad \phi_{s}(s, \omega, t)=0 \Leftrightarrow(s, \omega, t)=M \\
\phi_{s t}(M)<0, \quad \nabla_{s, \omega}\left(\phi_{s}\right)(M)=0, \quad \nabla_{s, \omega}^{2}\left(\phi_{s}\right)(M)>>0 .
\end{array}\right.
$$

The problem we want to solve in $D$ is

i) $\tilde{\mathcal{L}}(\lambda, \phi, v, w)=0$,

ii) $\phi$ satisfies $(\mathrm{H})$ in $D$.

We start the solving process with $\lambda=0$, taking for $\phi$ the approximate solution hinted at above. This approximate solution is essentially a solution of Burgers'equation with an initial datum $F_{0}$. Our assumption (ND) implies that this approximate $\phi$ precisely satisfies $(\mathrm{H})$. All we have to do then is to make sure that, when solving $\tilde{\mathcal{L}}=0$ by an iteration process, we can reproduce at each step this condition $(\mathrm{H})$.

Remark that condition $(\mathrm{H})$ will ensure that the singularity of $G$ is of cusp type (in the reduced variables). Since $\tilde{\mathcal{L}}$ comes from $\mathcal{L}$, its linearization has a special structure. With $q=\partial_{\lambda} \tau / \partial_{t} \tau$, we obtain (see [4]) that

$$
(\tilde{\mathcal{L}})_{(\lambda, \phi, v, w)}^{\prime}(\dot{\lambda}, \dot{\phi}, \dot{v}, \dot{w})=\dot{f}
$$

is equivalent to

$$
(\mathcal{L})_{\phi, v, w}^{\prime}(\dot{\Phi}, \dot{V}, \dot{W})=\dot{f}-q \dot{\lambda} \partial_{t} \tilde{\mathcal{L}}
$$

Here, by an abuse of notation, the left-hand side denotes the transform of $\mathcal{L}^{\prime}$ by the change of variables (2.1)

$$
(s, \omega, \tau) \rightarrow(s, \omega, t)
$$

The new unknowns are

$$
\dot{\Phi}=\dot{\phi}-\dot{\lambda} q \phi_{t}, \dot{V}=\dot{v}-\dot{\lambda} q v_{t}, \dot{W}=\dot{w}-\dot{\lambda} q w_{t}
$$

Assume now that, at some stage of an iteration process aimed at solving $\tilde{\mathcal{L}}=0$, the function $\phi$ satisfies $(H)$. We solve first (2.3), neglecting $\dot{\lambda} \partial_{t} \tilde{\mathcal{L}}$ in the right-hand side, since it is a quadratic error. We choose then $\dot{\lambda}$ such that

$$
\phi+\dot{\phi}=\phi+\dot{\Phi}+\dot{\lambda} q \phi_{t}
$$

again satisfies $(\mathrm{H})$ (with possibly a different point $M$ ). One easily sees that this is possible using the structure of $(\mathrm{H})$ and the implicit function theorem (see [4]). More precisely, we have the following lemma.

Lemme. Suppose $\phi^{(0)}$ satisfies condition $(\mathrm{H})$ for a point $\tilde{m}_{0}$. Assume that $\phi-\phi^{(0)}$ and $\psi$ are small enough in $C^{4}$. Then there exist a function $\Lambda(\phi, \psi)$ and a point $\tilde{m}(\phi, \psi)$ such that

$$
\Lambda\left(\phi^{(0)}, 0\right)=0, \quad \tilde{m}\left(\phi^{(0)}, 0\right)=\tilde{m}_{0}
$$

and the function $\phi+\psi+\Lambda(\phi, \psi) \partial_{t} \phi$ satisfies $(\mathrm{H})$ for the point $M=\left(\tilde{m}(\phi, \psi), t_{0}\right)$.

If we use a Nash-Moser iteration process, we have to take the smoothing process into account in this argument, since we solve the linearized system not exactly on $\phi+\dot{\phi}$, but on a smoothed approximation of it. The details can be found in [3]. 


\section{C.3. More on the geometry of the fixed domain $D$}

All we have said so far is valid assuming that we can understand the structure of the linearized blowup systems in the fixed domain $D$. As explained in B.3, this can be done if we are in a genuinely nonlinear situation, that is $\gamma \neq 0$ in $D$. In the model case $u_{t t}-\Delta u=u_{t} u_{t t}$ of [4], $\gamma$ is a non zero constant, we can take $D$ as in C.2. In the general situations of cases (1) or (2), however, we know that $\gamma=-g+o(1)$. All we know about $g$ comes from our assumption (ND). If $\left(\sigma_{0}, \omega_{0}\right)$ is the point where the maximum is reached, we know only $g\left(\omega_{0}\right) \neq 0$. Thus we have to shrink $D$ in such a way that $\omega$ remains close to $\omega_{0}$ in $D$. At the same time, $D$ has to remain an influence domain for the linearized blowup system. This can be arranged with a little care (see [5] for case (1) and [10] for case (2)).

The case (3) is more delicate, since one needs to know $\partial_{\sigma} G \neq 0$ in $D$ to be in a genuinely nonlinear situation. The way to attain this situation is to prove first that $u$ does not blowup in a strip corresponding to

$$
\sigma_{0}<\sigma_{1} \leq s \leq M, \quad \tau_{0} \leq \tau \leq \tau_{2}, \quad \tau_{2}>\bar{\tau},
$$

where $\sigma_{1}$ is close to $\sigma_{0}$. After that, we can essentially take for $D$ a domain for which

$$
\sigma_{-1} \leq s \leq \sigma_{1}, \quad 0 \leq t \leq t_{0}
$$

$\sigma_{-1}<\sigma_{0}$ being close enough to $\sigma_{0}$, with the correct shape. The details are rather tedious and can be found in [10].

\section{HOW TO SOLVE THE LINEARIZED BLOWUP SYSTEM}

We have seen in $\mathrm{C}$ how the problem of finding blowup solutions could be reduced to the problem of solving the (modified) linearized blowup system in a fixed domain $D$ with the correct shape. It is important here to notice that the blowup system has been linearized on a function $\phi$ satisfying $(H)$. At this stage of the proof, one should distinguish the three cases separately, since the linearized systems are a little different from each other. We details we give here correspond to the general case for $n=2$ (case (1) of I) handled in [4], [5]; the similar statements for the other cases can be found in [10].

Thus we have to solve a linear system of the form B.3 (3.1)-(3.2). Let us explain on the explicit case (3.3)-(3.4) the strategy used here to solve this coupled system. We set

$$
\dot{z}=Z_{0} \dot{k}, \quad P \equiv Z_{1} \partial_{s} Z_{1}-\phi_{s} Q Z_{1}+(Q \phi) \partial_{s} Z_{1}
$$

Then we rewrite (3.4) as

$$
Z_{1}\left(Z_{1} \dot{\phi}+Q \dot{k}\right)=\ldots
$$

Assuming that we have good energy inequalities for $P$ and $Z_{1}$, and using the smallness of the coupling coefficients $\alpha_{i}$, we obtain an energy inequality for the coupled system. Hence the problem is essentially to prove an inequality for $P$.

In the context of the present problem explained in $\mathrm{C}$, we have to single out what properties of $P$ are relevant. It turns out that the important features are 
i) The structure of $Z_{1}$,

ii) The sizes and signs of the coefficients of $Q$,

iii) The sizes of the coefficients of $Q_{1}$.

Simplifying a little bit here, we find

i) $Z_{1}=\left(-1+O\left(\epsilon^{2}\right)\right) \partial_{t}+O\left(\epsilon^{2}\right) \partial_{\omega}$,

ii) $Q=\epsilon^{2}\left(N_{1} Z_{1}^{2}+2 \epsilon^{2} N_{2} Z_{1} \partial_{\omega}+N_{3} \partial_{\omega}^{2}\right)$, with

$$
N_{1}=-n_{1}+O\left(\epsilon^{2}\right), \quad n_{3}=n_{3}+O\left(\epsilon^{2}\right), \quad n_{1}>0, \quad n_{3}>0,
$$

iii) $Q_{1}=O\left(\epsilon^{2}\right)$.

Since $P$ degenerates for $\phi_{s}=0$, we expect to obtain a weighted energy inequality. We set

$$
\delta=t_{0}-t, \quad g=\exp h(s-t), \quad p^{2}=\delta^{\mu} g, \quad|f|_{L^{2}(D)}=|f|_{0} .
$$

Here, $\mu>1$ is fixed, $h$ is a big parameter, and $\phi$ is assumed to satisfy $(\mathrm{H})$ and be close to some fixed $\phi^{(0)}$ in $C^{4}(D)$. We have then, assuming

$$
\dot{k}(s, \omega, 0)=\partial_{t} \dot{k}(s, \omega, 0)=0, \quad \dot{k}(M, \omega, t)=0,
$$

the following inequality:

$$
h\left|p \partial_{s} Z_{1} \dot{k}\right|_{0}^{2}+h\left|p Z_{1}^{2} \dot{k}\right|_{0}^{2}+\epsilon^{2} h\left|p \partial_{\omega} Z_{1} \dot{k}\right|_{0}^{2}+\epsilon^{4} \int \delta^{\mu-1} g \phi_{s}(1+\delta h)\left|\partial_{\omega}^{2} \dot{k}\right|^{2} \leq C|p P \dot{k}|_{0}^{2} .
$$

The proof of such an inequality is straightforward, once the good multiplier is found! Here we take

$$
M \dot{k}=-a \partial_{s} Z_{1} \dot{k}+\epsilon^{2} c \partial_{\omega}^{2} \dot{k}+d Z_{1}^{2} \dot{k}
$$

with

$$
a=\phi_{s}^{-1} \delta^{\mu} g, \quad c=c^{\prime} \delta^{\mu} g, \quad d=-d^{\prime} \delta^{\mu} g .
$$

Here, $c^{\prime}$ and $d^{\prime}$ are positive constants to be chosen. With this $M$, we compute

$$
\int_{D} P \dot{k} M \dot{k} d s d t d \omega
$$

It turns out that to ensure positivity of the boundary terms and of the quadratic form on $\partial^{2} \dot{k}$ we obtain by integrating by parts, we can take $d^{\prime}=1, c^{\prime}$ small enough. The further steps to complete the proof are standard, though tedious:

i) We commute with $P$ products of the fields $Z_{1}, \partial_{s}, \partial_{\omega}$. One has to check that the commutators can be absorbed in the left-hand side of the energy inequality.

ii) We deduce from the weighted $L^{2}$ control of such products tame estimates in the standard Sobolev norms. Remark that the weights in the energy inequality involve $\phi_{s}$, hence may change at each step: the obtention of estimates in Sobolev norms is important here since we have to work in a fixed scale of spaces.

iii) Existence is obtained for $P$ by truncation $P$ in the $\omega$ variables, and using the already proved estimates. 


\section{Bibliography}

[1] Alinhac S., "Explosion géométrique pour des systèmes quasi-linéaires", Amer. J. Math. 117(4), 1995, 987-1017.

[2] Alinhac S., "Temps de vie précisé et explosion géométrique pour des systèmes hyperboliques quasilinéaires en dimension un d'espace", Ann. Scuola Norm. Sup. Pisa, Serie IV vol. XXII (3), 1995, 493-515.

[3] Alinhac S., "Explosion des solutions d'une équation d'ondes quasi-linéaire en deux dimensions d'espace", Comm. PDE 21(5,6), 1996, 923-969.

[4] Alinhac S., "Blowup of small data solutions for a quasilinear wave equation in two space dimensions", Ann. Maths 149, 1999, 97-127.

[5] Alinhac S., "Blowup of small data solutions for a class of quasilinear wave equations in two space dimensions II", Acta Mat. 182, 1999, 1-23.

[6] Alinhac S., "Rank two singular solutions for quasilinear wave equations", Int. Res. Math. Notices 18, 2000, 955-984.

[7] Alinhac S., "Remarks on the blowup rate of classical solutions to quasilinear multidimensional hyperbolic systems", J. Math. Pure Appl. 79, 2000, 839-854.

[8] Alinhac S., "Stability of geometric blowup", Arch. Rat. Mech. Analysis 150, 1999, $97-125$.

[9] Alinhac S., "The null condition for quasilinear wave equations in two space dimensions I", Invent. Math. 145, (2001), 597-618.

[10] Alinhac S., "The null condition for quasilinear wave equations in two space dimensions II", Amer. J. Math. 123, (2000), 1-31.

[11] Alinhac S., "An Example of Blowup at Infinity for a Quasilinear Wave Equation", to appear in Astérisque.

[12] Alinhac S., "A remark on energy inequalities for perturbed wave equations", Preprint, Université Paris-Sud (Orsay), (2001).

[13] Alinhac S., "Blowup for nonlinear hyperbolic equations", Progress in Nonlinear Differential Equations and their Applications, Birkhäuser, Boston, 1995.

[14] Alinhac S. and Gérard P., "Opérateurs pseudo-différentiels et théorème de Nash-Moser", InterEditions, Paris, 1991.

[15] Christodoulou D. and Klainerman S., " The global nonlinear stability of the Minkowski space", Princeton Math. Series 41, (1993).

[16] Hörmander L., "Lectures on Nonlinear hyperbolic differential equations", Math. et Appl. 26, (1997), Springer Verlag. 
[17] Hoshiga A., "The initial value problems for quasilinear wave equations in two space dimensions with small data”, Adv. Math. Sci. Appl. 5, (1995), 67-89.

[18] Klainerman S., "Uniform decay estimates and the Lorentz invariance of the classical wave equation”, Comm. Pure Appl. Math. 38, (1985), 321-332.

[19] Klainerman S., "A Commuting Vectorfields Approach to Strichartz type Inequalities and Applications to Quasilinear Wave Equations", Int. Math. Res. Notices 5, (2001), 221-274.

[20] Klainerman S. and Sideris T., "On Almost Global Existence for Nonrelativistic Wave Equation in 3D", Comm. Pure Appl. Math. 49, (1996), 307-321.

[21] Kong De-xing, "Cauchy Problem for Quasilinear Hyperbolic Systems", Memoirs Math. Soc. Japan 6, (2000).

[22] Ladhari R., "Petites solutions d'équations d'ondes quasi-linéaires en dimension deux d'espace", Thèse de Doctorat, Université Paris-Sud, (1999).

[23] Sideris T., "The null condition and global existence of nonlinear elastic waves", Invent. Math. 123, (1996), 323-342.

\section{Contents}

Introduction

I QUASILINEAR WAVE EQUATIONS: CONCEPTS, RESULTS 2

$1 \quad$ Free solutions of the wave equation 2

2 The Z fields 3

$3 \quad$ Energy inequalities $\quad 4$

4 The function $g(\omega)$ and the null condition 6

$\begin{array}{llr}5 & \text { The slow time } & 7\end{array}$

$\begin{array}{llr}6 & \text { Reduced equations } & 8\end{array}$

II THE BLOWUP OF SMALL SOLUTIONS 9

$\begin{array}{ll}\text { A GEOMETRIC BLOWUP } & 10\end{array}$

$\begin{array}{lll}\text { A.1 Focusing and blowup in one space dimension } & 10\end{array}$ 
A.2 Geometric blowup in one space dimension 12

2.1 Some basic definitions . . . . . . . . . . . . . . . . . . . 12

2.2 Simple waves revisited . . . . . . . . . . . . . . . . . 14

A.3 Geometric blowup in multidimensional situations (systems) $\quad 14$

3.1 Blowup systems . . . . . . . . . . . . . . . . . . 14

3.1.a General definitions . . . . . . . . . . . . . . 14

3.1.b Some explanations about the choice of the blowup system . . 16

3.2 Blowup solutions, folds and cusps . . . . . . . . . . . . . . 17

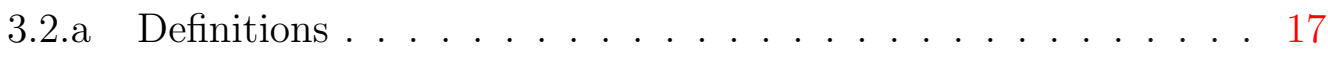

3.2.b Existence in the analytic case . . . . . . . . . . . 18

A.4 Geometric blowup in multidimensional situations (scalar second order equations)

B STRUCTURE OF THE LINEARIZED BLOWUP SYSTEMS

B.1 The linearized blowup system of a scalar second order equation 21

B.2 Geometric interpretation $\quad 22$

B.3 Decoupling of the linearized blowup system 22

B.4 How to solve the linearized blowup system 23

C REDUCTION TO A PROBLEM ON A FIXED COMPACT DOMAIN 24

C.1 The solution $u$ close to the boundary of the light cone 25

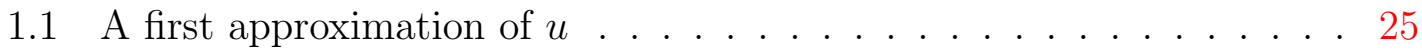

1.2 The function $G$ as a smooth function of its variables . . . . . . . . . . 25

C.2 The free boundary Goursat problem 26

2.1 The exact equation . . . . . . . . . . . . . . . 26

2.2 The stability lemma . . . . . . . . . . . . . . . 27

C.3 More on the geometry of the fixed domain $D \quad 29$

D HOW TO SOLVE THE LINEARIZED BLOWUP SYSTEM 29

Université PARIS-Sud, Orsay

Serge.Alinhac@math.u-psud.fr 